\title{
Salmonella enterica bacteraemia: a multi-national population-based cohort study
}

\author{
Kevin B Laupland*1, Henrik C Schønheyder², Karina J Kennedy³, Outi Lyytikäinen4, Louis Valiquette5, John Galbraith6, \\ Peter Collignon ${ }^{3}$ and the International Bacteremia Surveillance Collaborative
}

\begin{abstract}
Background: Salmonella enterica is an important emerging cause of invasive infections worldwide. However, population-based data are limited. The objective of this study was to define the occurrence of S. enterica bacteremia in a large international population and to evaluate temporal and regional differences.

Methods: We conducted population-based laboratory surveillance for all salmonella bacteremias in six regions (annual population at risk 7.7 million residents) in Finland, Australia, Denmark, and Canada during 2000-2007.

Results: A total of 622 cases were identified for an annual incidence of 1.02 per 100,000 population. The incidence of typhoidal (serotypes Typhi and Paratyphi) and non-typhoidal (other serotypes) disease was 0.21 and 0.81 per 100,000/ year. There was major regional and moderate seasonal and year to year variability with an increased incidence observed in the latter years of the study related principally to increasing rates of non-typhoidal salmonella bacteremias. Advancing age and male gender were significant risk factors for acquiring non-typhoidal salmonella bacteremia. In contrast, typhoidal salmonella bacteremia showed a decreasing incidence with advancing age and no gender-related excess risk.
\end{abstract}

Conclusions: Salmonella enterica is an important emerging pathogen and regional determinants of risk merits further investigation.

\section{Background}

Salmonella enterica is a major cause of invasive infections worldwide [1-7]. Although a wide range of serotypes may cause human disease, they may be broadly grouped into the typhoidal species that are specific human pathogens and includes serotypes Typhi and Paratyphi, and other serotypes that are primarily spread to humans from animal sources (non-typhoidal). In high income countries, a major risk factor for acquiring typhoidal salmonella bacteremia is travel to an endemic region [8,9]. Foreign travel is also a risk factor for acquiring non-typhoidal salmonella infections, and several reports have highlighted the spread of resistant species globally [10,11]. However, non-typhoidal salmonella may frequently cause human disease in high income countries and in these cases risk factors include exposure to contaminated food, extremes of age, and the presence of a number of co-morbid illnesses [12-16].

In order to best establish the distribution and determinants of an infectious disease, population-based studies are optimal. This is because in these designs, selection bias is minimized by inclusion of all cases of disease fulfilling a case definition occurring among residents of a defined population. In addition, because the population at risk can be defined, incidence rates can be calculated that facilitate comparison among different populations and time periods. Only a few, relatively small studies have investigated the epidemiology of S. enterica bacteremia at the population level in single regions [17-19]. The objective of this study was to define the occurrence of $S$. enterica bacteremia in a large international population and to evaluate temporal and regional differences.

\footnotetext{
* Correspondence: kevin.laupland@calgaryhealthregion.ca

1 Departments of Medicine and Pathology and Laboratory Medicine, University of Calgary and Calgary Laboratory Services, Calgary, Alberta, Canada Full list of author information is available at the end of the article
} 


\section{Methods}

\section{Study protocol and definitions}

This study utilized a multi-centre population-based cohort design. All incident episodes of $S$. enterica bacteremia as defined by growth from one or more blood cultures from a resident of the surveillance population during January 1, 2000 to December 31, 2007 were identified. Year of culture, patient's age and gender were available for all cases and further microbiology and clinical information such as admission status were collected from all sites except Finland.

Bacteremia was defined as either hospital-onset (first occurred $>2$ days after hospital admission) or community onset (first identified in community or within 2 days of hospital admission). Non-typhoidal salmonella infections were those exclusive of serotypes Typhi and Paratyphi. Only the first episode of salmonella bacteremia serotype per patient per year was included. Each center received approval from their local ethical review committee.

\section{Surveillance populations}

Surveillance was conducted in Denmark, Finland, Australia, and three regions in Canada under the auspices of the International Bacteremia Surveillance Collaborative [20]. Microbiology laboratory testing was performed by each region according to their local procedures. Data were abstracted from each region using a standardized template. The Danish surveillance region was the North Denmark Region using the previous boundaries of the North Jutland County (population 495,000). Data were obtained using the North Jutland Bacteremia Research Database administered by the Department of Clinical Microbiology at Aalborg Hospital [21]. Surveillance data from Finland (population 5.3 million) was obtained using the National Infectious Disease Register (NIDR) to which all Finnish clinical microbiology laboratories report all bacterial isolations from blood. The Canberra Region (population 370,000) includes the city of Canberra within the Australian Capital Territory and the satellite city of Queanbeyan and several small surrounding rural towns within the state of New South Wales. Surveillance was conducted in three of the four microbiology laboratories that are estimated to capture more than $95 \%$ of all positive blood cultures in the region [22]. The three Canadian centers included Sherbrooke, Quebec, Victoria, British Columbia, and Calgary, Alberta. Sherbrooke has a total population of 152,000 residents and is served by a single microbiology laboratory located in the Centre Hospitalier Universitaire de Sherbrooke [23]. Data from the Victoria area included the south local health area of the Vancouver Island Health Authority (population 364,000), and data were obtained from the regional microbiology laboratory [20]. Laboratory based surveillance in the Calgary Health Region (population 1.2 million) was conducted at
Calgary Laboratory Services that performs virtually all of the blood culture testing among residents of the region [24].

\section{Data management and statistical analysis}

Data from each of the surveillance centres was compiled and analysed using Stata version 10.0 (Stata Corp, College Station, TX). Prior to description of analysis, all continuous variables were plotted using histograms to assess their underlying distribution. Non-normally distributed variables were reported as medians with inter-quartile ranges (IQR). The incidence of salmonella bacteremia was calculated by dividing the number of incident cases by the surveillance population as determined by census data from each of the surveillance areas. Incidence rates overall and for non-typhoidal and typhoidal salmonella bacteremia were directly standardized by age deciles and gender to the 2007 27-country European Union (EU27) population and reported as rates per 100,000 inhabitants [20]. Because of the small numbers in many cells, crude incidence rates were reported in sub-group analyses. Risks in population groups were expressed as incidence rate ratios (RR) and reported with 95\% confidence intervals.

\section{Results}

During the 8-year study, a total of 622 incident S. enterica bacteremias occurred during 61,748,478 person-years of surveillance; 370 (59\%), 107 (17\%), 78 (13\%), 27 (4\%), 21 (3\%) and 19 (3\%) cases were from Finland, Calgary, North Denmark, Sherbrooke, Victoria, and Canberra, respectively. Non-typhoidal isolates accounted for 490 (79\%) cases, and of the 132 typhoidal salmonella cases, 85 (64\%) were serotype Typhi and 47 (36\%) were serotype Paratyphi.

\section{Incidence}

The overall age and gender standardized annual incidence rate was 1.02 per 100,000 population, and was 0.21 and 0.81 per 100,000 for typhoidal and non-typhoidal bacteremia, respectively. The adjusted incidence rates were markedly different among the regions and by salmonella group as shown in Figure 1. There was moderate year to year variability with an overall increase in crude incidence observed in the latter years of the study related principally to increasing rates of non-typhoidal salmonella bacteremia as shown in Figure 2. The occurrence of salmonella bacteremia varied during the months of the year with the highest frequency in the late summer and early fall months as shown in Figure 3.

\section{Demographic factors}

The overall risk of acquiring salmonella bacteremia varied significantly by age, gender, and salmonella group as shown in Figures 4a and 4b. Among non-typhoidal sal- 


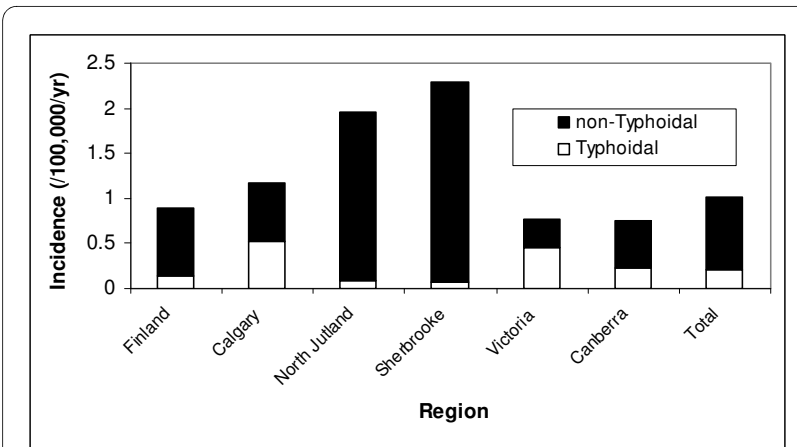

Figure $1 \mathrm{Age}$ and gender standardized incidence of salmonella bacteremia by region and group, 2000-2007.

monella infections (Figure 4a), the risk increased with advancing age and males were at significantly higher risk (RR 1.62; 95\% CI, 1.35-1.96; $\mathrm{p}<0.0001$ ). The excess risk for non-typhoidal infections in males was most notable among adults aged 70 to 89 (Figure 4a), with the markedly increased incidence of non-typhoidal salmonella bacteremia observed among aged 90 and older attributable to an excess number of cases in one centre (Denmark). In contrast, typhoidal salmonella bacteremias (Figure 4b) showed a decreasing incidence with advancing age and males were not at increased risk (RR 1.14; 95\% CI, 0.80$1.62 ; \mathrm{p}=0.47)$.

\section{Clinical and microbiologic information}

Further clinical and microbiology information was available for 252 cases. Among these, 234 (93\%) were classified as community-onset and 204 (81\%) were admitted to hospital for a median length of stay of 7 days (IQR, 4-11 days). Of the 18 hospital-onset cases, all were nontyphoidal serotypes and $10(56 \%)$ were isolated during the first 3-5 days of admission. Of the remaining eight, the onset was 6, 8, 9 (2 cases), 15, 21 (2 cases) and 25 days after admission.

Among the 252 cases with added details, 55 (22\%) were serotype Typhi, 20 (8\%) were serotype Paratyphi, and 177

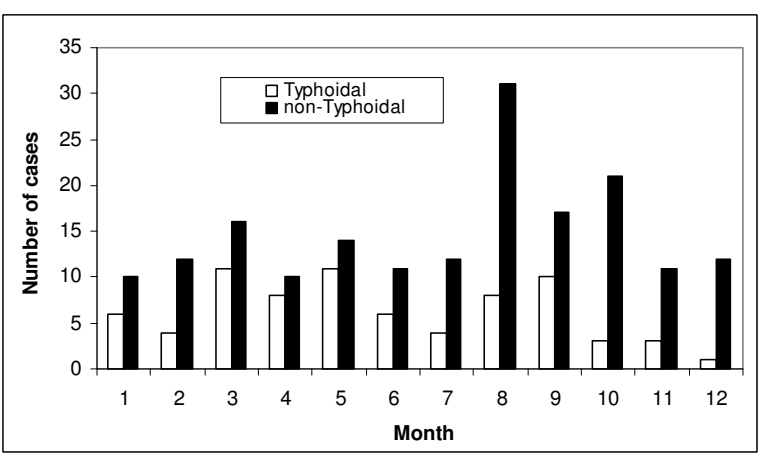

Figure 2 Annual incidence of salmonella bacteremia, 2000-2007.

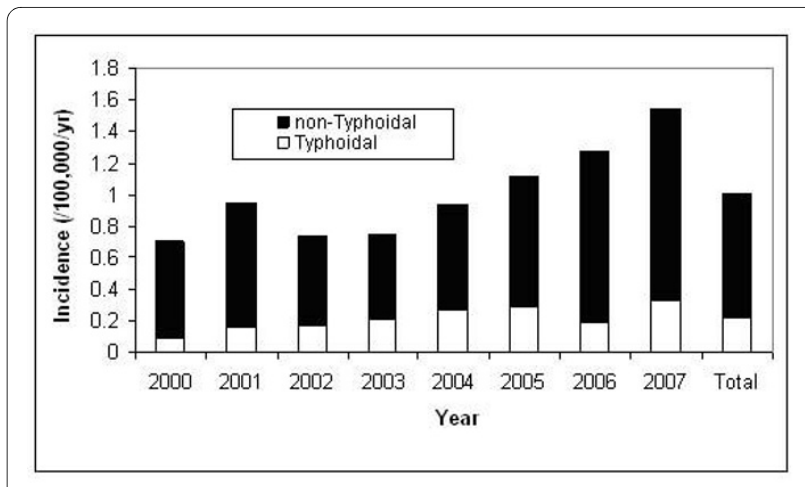

Figure 3 Monthly occurrence of salmonella bacteremia (data from Finland not included). Month shown refers to month of the year (ie January = month 1) for northern hemisphere sites and adjusted by 6 months for opposite season in Australia (ie January = month 7).

(70\%) were non-typhoidal serotypes that included $36 S$. Enteritidis, $22 \mathrm{~S}$. Heidelberg, $20 \mathrm{~S}$. Typhimurium, $8 \mathrm{~S}$. Newport, 7 each of $S$. Dublin and $S$. Virchow, $6 S$. Hadar, 4 each of $S$. Oranienburg and $S$. Saint Paul, 3 each of $S$. Java, S. Panama, S. Poona, S. Choleraesuis, and $S$. San Diego, $2 S$. Arizonae, and one each of $S$. Muenster, $S$. Infantis, $S$. Matadi, $S$. Schwarzengrund, S. Bournemouth, $S$. Napoli, $S$. Bovismorbificans, $S$. Colindale, $S$. Haifa, $S$. Corvallis and $S$. Agona. Other isolates not identified to

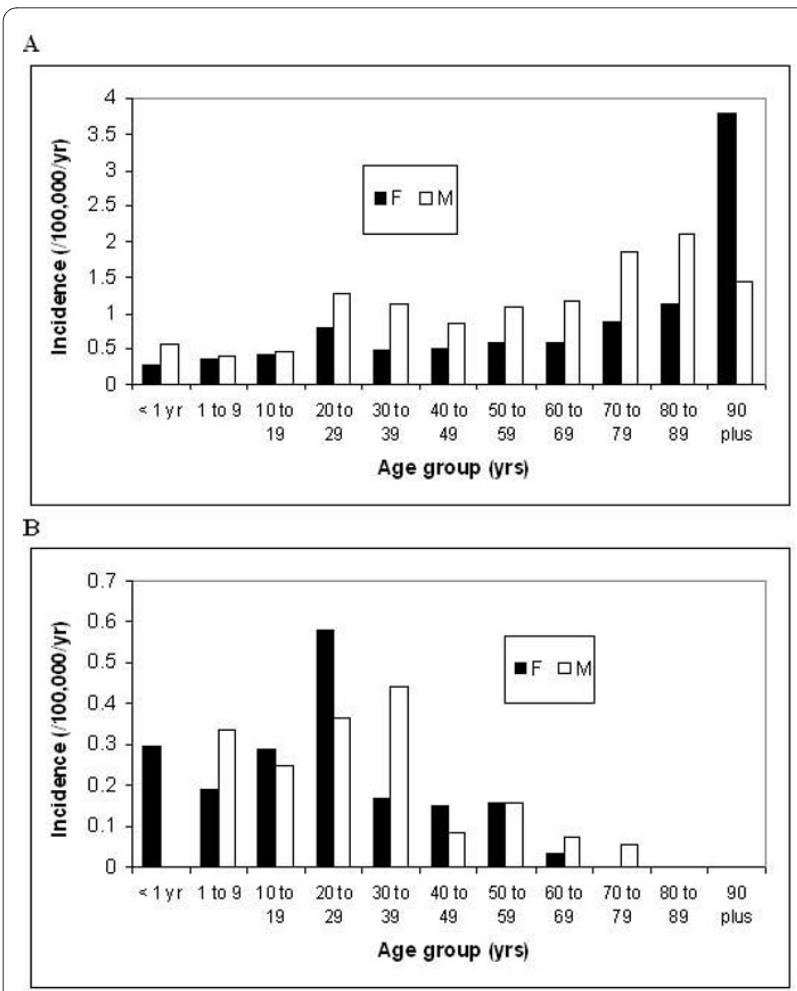

Figure 4 Age and gender specific incidence of a) non-typhoidal and b) typhoidal salmonella bacteremia, 2000-2007. 
the specific serotype level included salmonella group $\mathrm{C} 1 /$ 2 in 8 , group D in 4 , group B in 18 , and were not reported in 5 cases.

Susceptibility testing results were available for 246 isolates. Reduced susceptibility to ampicillin was found in 52 (21\%), and to ciprofloxacin in 7 (3\%), and ceftriaxone in 7 (3\%) cases. All isolates with reduced susceptibility to ceftriaxone were from Sherbrooke and 6/7 ciprofloxacin resistant isolates were from North Denmark.

\section{Discussion}

This study reports the first novel results of the evolving International Bacteremia Surveillance Collaborative and provides new population-based information on the epidemiology of salmonella bacteremia. Our collaborative was initially formed in 2008 to address the lack of coordinated population based study of invasive bacterial diseases internationally [20]. Our present results highlight the value of such an initiative. The more than 60 million combined patient years of observation enabled us to study a large number of cases in low incidence areas. In addition, we were able to directly calculate the incidence of disease because in our surveillance areas all cases were included (sampling was not performed) and we had welldefined populations at risk for denominator data. This contrasts with non-population based studies such as hospital-based case series where sampling bias is a potentially major concern and the population at risk is rarely definable for community-onset disease [25]. Furthermore, by age and gender standardization to the EU27 population, we were able to meaningfully compare rates of disease in our surveillance regions. We observed that salmonella bacteremia is a relatively uncommon cause of bacteremia in our populations, but that considerable demographic and regional differences in occurrence exist.

The observations of significant differences among regions and by species group are important and novel. While we believe these represent true differences in incidence, a number of other factors could potentially at least in part explain these observations. Since the risk for salmonella bacteremia is influenced by age and gender, where populations differ in demographic structure then crude incidence rates may be expected to differ. However, we controlled for this possibility by age and gender standardizing our overall incidence rates. A second potential issue is that there may have been different rates of case ascertainment (identifying all culture-positive cases among residents) among our surveillance regions. While in some cases ascertainment was expected to approach or equal $100 \%$ such as with the national mandatory reporting system in Finland, this was not strictly the case in every other region [20]. However, all the other surveillance areas in this study are highly captive and estimated to be $95 \%$ or higher, such that the $2-3$ fold differences in rates could not be explained by these small potential differential rates of ascertainment. A third possibility is that there may have been differences in the culturing practices among regions. This arises if the decision to sample blood for culture is significantly different among regions, and it may be expected that areas that sample more frequently may observe higher numbers of culture-positive cases. It is important to note that in all of our study regions the cost of blood culturing is fully publicly funded and available to all residents irrespective of financial means. While we cannot directly assess the issue of differential blood culturing pratices among our regions as we do not have data on negative cultures, it is notable that in previous individual publications on other common species causing bloodstream infections such as Escherichia coli and Staphylococcus aureus, similar crude incidence rates were reported [22-24,26,27]. Although we may speculate as to why the incidence of salmonella bacteremia differs so greatly among our regions, further studies are needed to explore factors such as travel, food preparation practices, agricultural, and other factors $[8,28]$.

Our observation of increased risk for salmonella bacteremia during the summer has been previously well documented $[17,29,30]$, and has been proposed to be due to increased travel abroad and lax food preparation related to outdoor cooking in that season. Several previous studies have documented increased colonization rates with salmonella species among food animals during the summer months [31-33]. Our observation of an increased risk for non-typhoidal salmonella bacteremia with advancing age is likely at least in part due to a higher prevalence of co-morbid illnesses in the older age group that predispose to bacteremia risk [25,34]. The excess population risk in males for non-typhoidal salmonella bacteremia is a novel finding. We speculate that this may be due to agricultural or diet exposure in males. Typhoidal salmonella bacteremia occurred in younger individuals and no gender-associated risk was evident. We suspect that this may reflect a greater rate of foreign travel to exotic typhoidal salmonella endemic regions in the younger age groups or possibly may reflect younger immigrant populations. However, while we speculate reasons as to why age and gender may modify risk, we do not have empiric data to support these suspicions. The determinants of increased risk for salmonella bacteremia in our surveillance regions merits further prospective investigation.

While this study benefits from the large, multi-national, population-based design, there are some limitations that merit discussion. The surveillance regions involved in this study are high income Western countries with largely publicly funded health systems. These results should therefore not be generalized to other dissimilar jurisdictions especially low income countries and tropical areas. 
In low income countries salmonella typically is among the most common bacterial pathogens and may be responsible for as many as one-half of all bacteremias $[6,35,36]$ whereas they typically represent $\leq 1 \%$ in high income countries [18,37]. Incidence rates for typhoid in the developing world, especially the Indian subcontinent, may be several orders of magnitude higher than that reported here $[7,36]$. Second, the information that we collected was retrospective. While we used common criteria for inclusion procedures and data were abstracted using a standardized template, the routine laboratory testing procedures and methods of clinical data collection varied somewhat site-by-site. For example, only basic information was available from Finnish cases, and microbiology and clinical details from the other centers were determined using the local data sets and procedures. These included prospective review in Denmark and electronic records review and/or database extraction in other sites. In addition, ideally isolates would have been serotyped and tested for antimicrobial susceptibility in a single centralized laboratory. Finally, it would have been useful to have added information on other potential risk factors such as food and animal exposures, international travel, and co-morbid illness.

\section{Conclusions}

In summary, in this multi-national collaborative study we report the epidemiology of salmonella bacteremia and find regional and temporal differences in occurrence. Further studies designed to define the determinants of risk for acquiring salmonella bacteremia are warranted. This report highlights the benefit of international cooperation in bacteremia surveillance and sets the stage for future collaborative studies.

\section{Competing interests}

The authors declare that they have no competing interests.

\section{Authors' contributions}

$\mathrm{KBL}$ contributed to data collection and conducted the primary analysis and drafting of the manuscript. HCS, KJK, OL, LV, JG, and PC contributed to data collection and manuscript preparation. All authors read and approved the manuscript.

\section{Acknowledgements}

All members of the International Bacteremia Surveillance Collaborative (Kevin B. Laupland, Deirdre L. Church, Daniel B. Gregson, Calgary, Canada; John Galbraith, Pamela Kibsey, Victoria Canada; Louis Valiquette, Sherbrooke, Canada; Henrik C. Schonheyder, Aalborg, Denmark; Jenny Dahl Knudsen, Christian. Ostergaard Andersen, Copenhagen, Denmark; Outi Lyytikainen, Helsinki, Finland, and Karina J. Kennedy, Peter Collignon, Canberra, Australia) contributed to protocol design and approval of the final manuscript. We thank Paul Whiting, Director of Clinical Pathology, Capital Pathology, Canberra Australia for assistance with case identification. No external funding was received in support of this study.

\section{Author Details}

'Departments of Medicine and Pathology and Laboratory Medicine, University of Calgary and Calgary Laboratory Services, Calgary, Alberta, Canada, 2Department of Clinical Microbiology, Aalborg Hospital, Aarhus University Hospital, Aalborg, Denmark, ${ }^{3}$ nfectious Diseases Unit and Microbiology Department, The Canberra Hospital and School of Clinical Medicine, Australian National University, Woden, Australian Capital Territory, Australia, ${ }^{4}$ Department of Infectious Disease Epidemiology, Hospital Infection Program, National Public Health Institute, Helsinki, Finland, ${ }^{5}$ Department of Microbiology-Infectious Diseases, Université de Sherbrooke, Sherbrooke, Québec, Canada and ${ }^{6}$ Microbiology Laboratory, Vancouver Island Health Authority, Royal Jubilee Hospital, Victoria, British Columbia, Canada

Received: 11 January 2010 Accepted: 14 April 2010 Published: 14 April 2010

\section{References}

1. Nielsen H, Gradel KO, Schonheyder HC: High incidence of intravascular focus in nontyphoid Salmonella bacteremia in the age group above 50 years: a population-based study. Apmis 2006, 114(9):641-645.

2. Kariuki S, Revathi G, Kiiru J, Lowe B, Berkley JA, Hart CA: Decreasing prevalence of antimicrobial resistance in non-typhoidal Salmonella isolated from children with bacteraemia in a rural district hospital, Kenya. Int J Antimicrob Agents 2006, 28(3):166-171.

3. Enwere G, Biney E, Cheung YB, Zaman SM, Okoko B, Oluwalana C, Vaughan A, Greenwood B, Adegbola R, Cutts FT: Epidemiologic and clinical characteristics of community-acquired invasive bacterial infections in children aged 2-29 months in The Gambia. Pediatr Infect Dis J 2006, 25(8):700-705.

4. Herz AM, Greenhow TL, Alcantara J, Hansen J, Baxter RP, Black SB, Shinefield HR: Changing epidemiology of outpatient bacteremia in 3to 36-month-old children after the introduction of the heptavalentconjugated pneumococcal vaccine. Pediatr Infect Dis J 2006, 25(4):293-300.

5. Broide E, Shapiro M, Boldur I, Klinowski E, Kimchi AN, Gluskin Y, Scapa E: Salmonellosis: an epidemiologic study. Isr Med Assoc J 2005, 7(2):91-94.

6. Berkley JA, Lowe BS, Mwangi I, Williams T, Bauni E, Mwarumba S, Ngetsa C, Slack MP, Njenga S, Hart CA, et al:: Bacteremia among children admitted to a rural hospital in Kenya. N Eng/ J Med 2005, 352(1):39-47.

7. Sinha A, Sazawal S, Kumar R, Sood S, Reddaiah VP, Singh B, Rao M, Naficy A, Clemens JD, Bhan MK: Typhoid fever in children aged less than 5 years. Lancet 1999, 354(9180):734-737.

8. Mermin JH, Townes JM, Gerber M, Dolan N, Mintz ED, Tauxe RV: Typhoid fever in the United States, 1985-1994: changing risks of international travel and increasing antimicrobial resistance. Arch Intern Med 1998, 158(6):633-638.

9. Ackers ML, Puhr ND, Tauxe RV, Mintz ED: Laboratory-based surveillance of Salmonella serotype Typhi infections in the United States: antimicrobial resistance on the rise. Jama 2000, 283(20):2668-2673.

10. Kariuki S, Revathi G, Kariuki N, Kiiru J, Mwituria J, Muyodi J, Githinji JW, Kagendo D, Munyalo A, Hart CA: Invasive multidrug-resistant nontyphoidal Salmonella infections in Africa: zoonotic or anthroponotic transmission? J Med Microbiol 2006, 55(Pt 5):585-591.

11. Helms M, Ethelberg S, Molbak K: International Salmonella Typhimurium DT104 infections, 1992-2001. Emerg Infect Dis 2005, 11(6):859-867.

12. Puthucheary SD, Ng KP, Hafeez A, Raja NS, Hassan HH: Salmonellosis in persons infected with human immunodeficiency virus: a report of seven cases from Malaysia. Southeast Asian J Trop Med Public Health 2004, 35(2):361-365.

13. Abramson S, Kramer SB, Radin A, Holzman R: Salmonella bacteremia in systemic lupus erythematosus. Eight-year experience at a municipal hospital. Arthritis Rheum 1985, 28(1):75-79.

14. Chen JY, Luo SF, Wu YJ, Wang CM, Ho HH: Salmonella septic arthritis in systemic lupus erythematosus and other systemic diseases. Clin Rheumatol 1998, 17(4):282-287.

15. Fang FC, Fierer J: Human infection with Salmonella dublin. Medicine (Baltimore) 1991, 70(3):198-207. 
16. Hand WL, King NL: Deficiency of serum bactericidal activity against Salmonella typhimurium in sickle cell anaemia. Clin Exp Immunol 1977, 30(2):262-270.

17. Gradel KO, Dethlefsen C, Schonheyder HC, Ejlertsen T, Sorensen HT, Thomsen RW, Nielsen $\mathrm{H}$ : Severity of infection and seasonal variation of non-typhoid Salmonella occurrence in humans. Epidemiol Infect 2007 135(1):93-99.

18. Gradel KO, Schonheyder HC, Pedersen L, Thomsen RW, Norgaard M, Nielsen $\mathrm{H}$ : Incidence and prognosis of non-typhoid Salmonella bacteraemia in Denmark: a 10-year county-based follow-up study. Eur J Clin Microbiol Infect Dis 2006, 25(3):151-158.

19. Yagupsky P, Maimon N, Dagan R: Increasing incidence of nontyphi Salmonella bacteremia among children living in southern Israel. Int $\mathrm{J}$ Infect Dis 2002, 6(2):94-97.

20. Laupland KB, Schonheyder HC, Kennedy KJ, Lyytikainen O, Valiquette L, Galbraith J, Collignon P, Church DL, Gregson DB, Kibsey P: Rationale for and protocol of a multi-national population-based bacteremia surveillance collaborative. BMC Research Notes 2009, 2:146.

21. Madsen KM, Schonheyder HC, Kristensen B, Sorensen HT: Secular trends in incidence and mortality of bacteraemia in a Danish county 19811994. Apmis 1999, 107(3):346-352.

22. Kennedy KJ, Roberts JL, Collignon PJ: Escherichia coli bacteraemia in Canberra: incidence and clinical features. Med J Aust 2008 188(4):209-213.

23. Allard C, Carignan A, Bergevin M, Boulais I, Tremblay V, Robichaud P, Duperval R, Pepin J: Secular changes in incidence and mortality associated with Staphylococcus aureus bacteraemia in Quebec Canada, 1991-2005. Clin Microbiol Infect 2008, 14(5):421-428.

24. Laupland KB, Gregson DB, Church DL, Ross T, Pitout JD: Incidence, risk factors and outcomes of Escherichia coli bloodstream infections in a large Canadian region. Clin Microbiol Infect 2008, 14(11):1041-1047.

25. Rodriguez M, de Diego I, Martinez N, Rosario Rodicio M, Carmen Mendoza M: Nontyphoidal Salmonella causing focal infections in patients admitted at a Spanish general hospital during an 11-year period (19912001). Int J Med Microbiol 2006, 296(4-5):211-222.

26. Lyytikainen O, Ruotsalainen E, Jarvinen A, Valtonen V, Ruutu P: Trends and outcome of nosocomial and community-acquired bloodstream infections due to Staphylococcus aureus in Finland, 1995-2001. Eur J Clin Microbiol Infect Dis 2005, 24(6):399-404

27. Laupland KB, Ross T, Gregson DB: Staphylococcus aureus bloodstream infections: risk factors, outcomes, and the influence of methicillin resistance in Calgary, Canada, 2000-2006. J Infect Dis 2008, 198(3):336-343

28. Ispahani P, Slack RC: Enteric fever and other extraintestinal salmonellosis in University Hospital, Nottingham, UK, between 1980 and 1997. Eur J Clin Microbiol Infect Dis 2000, 19(9):679-687.

29. Biendo M, Laurans G, Thomas D, Dechepy O, Hamdad-Daoudi F, Canarelli $B, E b$ F: Regional dissemination of Salmonella enterica serovar Enteritidis is season dependent. Clin Microbiol Infect 2003, 9(5):360-369.

30. Gordon MA, Walsh AL, Chaponda M, Soko D, Mbvwinji M, Molyneux ME, Gordon SB: Bacteraemia and mortality among adult medical admissions in Malawi--predominance of non-typhi salmonellae and Streptococcus pneumoniae. J Infect 2001, 42(1):44-49.

31. Hald T, Andersen JS: Trends and seasonal variations in the occurrence of Salmonella in pigs, pork and humans in Denmark, 1995-2000. Berl Munch Tierarzt/ Wochenschr 2001, 114(9-10):346-349.

32. Michel P, Martin LJ, Tinga CE, Dore K: Regional, seasonal, and antimicrobial resistance distributions of salmonella typhimurium in Canada: a multi-provincial study. Can J Public Health 2006, 97(6):470-474.

33. Fossler CP, Wells SJ, Kaneene JB, Ruegg PL, Warnick LD, Bender JB, Eberly LE, Godden SM, Halbert LW: Herd-level factors associated with isolation of Salmonella in a multi-state study of conventional and organic dairy farms II. Salmonella shedding in calves. Prev Vet Med 2005, 70(34):279-291

34. Shimoni Z, Pitlik S, Leibovici L, Samra Z, Konigsberger H, Drucker M, Agmon V, Ashkenazi S, Weinberger M: Nontyphoid Salmonella bacteremia: age-related differences in clinical presentation, bacteriology, and outcome. Clin Infect Dis 1999, 28(4):822-827.

35. Gordon MA, Graham SM, Walsh AL, Wilson L, Phiri A, Molyneux E, Zijlstra EE, Heyderman RS, Hart CA, Molyneux ME: Epidemics of invasive Salmonella enterica serovar enteritidis and S. enterica Serovar typhimurium infection associated with multidrug resistance among adults and children in Malawi. Clin Infect Dis 2008, 46(7):963-969.

36. Brooks WA, Hossain A, Goswami D, Nahar K, Alam K, Ahmed N, Naheed A, Nair GB, Luby S, Breiman RF: Bacteremic typhoid fever in children in an urban slum, Bangladesh. Emerg Infect Dis 2005, 11(2):326-329.

37. Laupland KB, Gregson DB, Flemons WW, Hawkins D, Ross T, Church DL: Burden of community-onset bloodstream infection: a populationbased assessment. Epidemiol Infect 2007, 135(6):1037-1042.

\section{Pre-publication history}

The pre-publication history for this paper can be accessed here: http://www.biomedcentral.com/1471-2334/10/95/prepub

\section{doi: 10.1186/1471-2334-10-95}

Cite this article as: Laupland et al., Salmonella enterica bacteraemia: a multinational population-based cohort study BMC Infectious Diseases 2010, 10:95

\section{Submit your next manuscript to BioMed Central and take full advantage of:}

- Convenient online submission

- Thorough peer review

- No space constraints or color figure charges

- Immediate publication on acceptance

- Inclusion in PubMed, CAS, Scopus and Google Scholar

- Research which is freely available for redistribution
C Biomed Central 